\title{
Highly effective SNP-based association mapping and management of recessive defects in livestock
}

\author{
Carole Charlier ${ }^{1}$, Wouter Coppieters ${ }^{1}$, Frédéric Rollin ${ }^{2}$, Daniel Desmecht ${ }^{3}$, Jorgen S Agerholm ${ }^{4}$, \\ Nadine Cambisano $^{1}$, Eloisa Carta ${ }^{5}$, Sabrina Dardano ${ }^{6}$, Marc Dive, Corinne Fasquelle ${ }^{1}$, Jean-Claude Frennet, \\ Roger Hanset ${ }^{1,11}$, Xavier Hubin ${ }^{7}$, Claus Jorgensen ${ }^{8}$, Latifa Karim ${ }^{1}$, Matthew Kent ${ }^{9}$, Kirsten Harvey ${ }^{5}$, \\ Brian R Pearce ${ }^{5}$, Patricia Simon ${ }^{1}$, Nico Tama ${ }^{1}$, Haisheng Nie ${ }^{1,10}$, Sébastien Vandeputte ${ }^{2}$, Sigbjorn Lien ${ }^{9}$, \\ Maria Longeri ${ }^{6}$, Merete Fredholm ${ }^{8}$, Robert J Harvey ${ }^{5} \&$ Michel Georges $^{1}$
}

\begin{abstract}
The widespread use of elite sires by means of artificial insemination in livestock breeding leads to the frequent emergence of recessive genetic defects, which cause significant economic and animal welfare concerns. Here we show that the availability of genome-wide, high-density SNP panels, combined with the typical structure of livestock populations, markedly accelerates the positional identification of genes and mutations that cause inherited defects. We report the fine-scale mapping of five recessive disorders in cattle and the molecular basis for three of these: congenital muscular dystony (CMD) types 1 and 2 in Belgian Blue cattle and ichthyosis fetalis in Italian Chianina cattle. Identification of these causative mutations has an immediate translation into breeding practice, allowing marker assisted selection against the defects through avoidance of at-risk matings.
\end{abstract}

Livestock productivity has improved remarkably over the last 50 years, to a large extent because of efficient breeding programs rooted in quantitative genetics. However, although stringent selection increases profitability, it may cause drastic reductions in effective population size, particularly when artificial insemination is heavily relied upon. For example, although the Holstein-Friesian dairy cattle population numbers several million individuals worldwide, its effective population size is in the low hundreds ${ }^{1,2}$. Because of the accompanying increase in coancestry and inbreeding, the industry faces regular outbreaks of recessive defects. For instance, in the 1990 s, $\sim 14 \%$ of Holstein-Friesian bulls were shown to carry a missense mutation in gene encoding the leukocyte $\beta 2$ integrin subunit CD18, causing bovine leukocyte adhesion deficiency (BLAD). This lethal immuno- deficiency affected $\sim 0.2 \%$ of newborn calves, at an estimated annual cost in the United States of $\$ 5$ million $^{3}$. More recently, $\sim 25 \%$ of Holstein-Friesian bulls were estimated to be carriers of a missense mutation in the gene encoding the Golgi UDP- $N$-acetylglucosamine transporter SLC35A3, causing complex vertebral malformation $(\mathrm{CVM})^{4}$. Genealogical studies determined that both genetic defects were likely disseminated in the population by a founder sire of the modern Holstein-Friesian breed: Carlin-M Ivanhoe Bell ${ }^{3,4}$. It is crucial that the breeding industry implement effective strategies to control such outbreaks. A path toward that goal is to establish surveillance centers to detect emerging defects and centralize samples of affected animals that can then be used to map and identify causal mutations. The ensuing diagnostic markers could then be used to avoid at-risk matings.

Toward that goal, we collected samples from affected animals for five emerging defects in four cattle breeds: congenital muscular dystonia 1 (CMD1), congenital muscular dystonia 2 (CMD2) and crooked tail syndrome (CTS) in Belgian Blue cattle, renal lipofuscinosis (RL) in Holstein-Friesian and Danish Red cattle and ichthyosis fetalis (IF) in Italian Chianina cattle. All calves with CMD have episodes of generalized muscle contractures, but careful clinical examination suggested two distinct phenotypes (CMD1 and CMD2). Calves affected by CMD1 show impaired swallowing, fatigue upon stimulation or exercise, and muscle myotonia resulting in an inability to flex limbs and injurious falling. CMD1 calves usually die within a few weeks as a result of respiratory complications. CMD2 calves suffer severe episodes of myoclonus upon acoustic or tactile stimulation and typically die within a few hours of birth. CMD2 is reminiscent of congenital myoclonus in Hereford cattle caused by a

${ }^{1}$ Unit of Animal Genomics, GIGA-Research and Department of Animal Sciences, Faculty of Veterinary Medicine University of Liège (B34), 1 Avenue de I'Hôpital, 4000 Liège, Belgium. ${ }^{2}$ Department of Large Animal Internal Medicine and ${ }^{3}$ Unit of Pathology, Department of Morphology and Pathology, Faculty of Veterinary Medicine, University of Liège, 20 Boulevard de Colonster, 4000 Liège, Belgium. ${ }^{4}$ Department of Veterinary Pathobiology, Faculty of Life Sciences, University of Copenhagen, 15 Groennegaardsvej, 1870 Frederiksberg C, Denmark. ${ }^{5}$ Department of Pharmacology, The School of Pharmacy, $29-39$ Brunswick Square, London WC1N 1AX, UK. ${ }^{6}$ Department of Animal Science, Faculty of Veterinary Medicine, University of Milan, 10 Via Celoria, 20133 Milan, Italy. ${ }^{7}$ Recherche \& Développement, Association Wallonne de l'Elevage, 4 Rue des Champs Elysées, 5590 Ciney, Belgium. ${ }^{8}$ Division of Genetics and Bioinformatics, Department of Animal and Veterinary Basic Sciences, Faculty of Life Sciences, University of Copenhagen, 3 Groennegaardsvej, 1870 Frederiksberg C, Denmark. ${ }^{9}$ Centre for Integrative Genetics, Department of Animal and Aquacultural Sciences, Norwegian University of Life Sciences, PO Box 5003, 1432 Aas, Norway. ${ }^{10}$ Present address: Animal Breeding and Genetics Group, Wageningen University, Wageningen, The Netherlands. 11Deceased. Correspondence should be addressed to M.G. (michel.georges@ulg.ac.be). 
Table 1 Overview of mapping studies

\begin{tabular}{|c|c|c|c|c|c|c|c|}
\hline \multirow[b]{2}{*}{ Defect } & \multicolumn{3}{|c|}{ Population } & \multicolumn{3}{|c|}{ Mapping } & \multirow[b]{2}{*}{ Gene } \\
\hline & Breed & Cases $^{a}$ & Controls ${ }^{a}$ & $\log (1 / p)^{\mathrm{b}}$ & Chrom. & Interval & \\
\hline Congenital muscular dystonia 2 (CMD2) & Belgian Blue & $7(21)$ & $24(2,000)$ & $>4^{c}$ & 29 & $3.61 \mathrm{Mb}$ & SLC6A5 \\
\hline Ichthyosis fetalis (IF) & Chianina & $3(3)$ & $9(96)$ & $3.30^{d}$ & 2 & $11.78 \mathrm{Mb}$ & $A B C A 12$ \\
\hline Crooked tail syndrome (CTS) & Belgian Blue & $8(36)$ & $14(2,000)$ & $>4^{c}$ & 19 & $2.42 \mathrm{Mb}$ & - \\
\hline
\end{tabular}

aNumbers correspond to sample sizes used to perform the genome-wide scan, whereas the numbers in brackets correspond to the total number of samples available. ${ }^{b} \mathrm{Highest}$ genome-wide log(1/p) value obtained using either ASSHOMc or ASSIST ${ }^{d}$. -, gene not known.

nonsense mutation in the gene encoding the $\alpha 1$ subunit of the inhibitory glycine receptor ${ }^{5}$. Despite the high incidence of CMD in Belgian Blue cattle $(0.1-0.2 \%)$, the distinction between the two forms, as well as their inherited nature, has remained controversial ${ }^{6}$. CTS is an emerging syndrome in Belgian Blue cattle. Affected animals have a crooked tail and shortened head, growth retardation, extreme muscularity and spastic paresia, although some characteristics show variable penetrance. CTS is not lethal per se, but causes substantial economic losses due to growth retardation and treatment. $\mathrm{RL}$ is characterized by the accumulation of lipofuscine granules in secondary lysosomes of renal proximal tubular epithelial cells, as well as in macrophages and in the reticular and endothelial cells of kidney-associated lymph nodes. Affected animals are identified at slaughter by their discolored kidneys, justifying the more common designation of 'black kidney disease'. RL is not accompanied by gross renal dysfunction, yet it may cause more insidious deleterious effects, as evidenced by a reduced longevity of affected

Figure 1 Congenital muscular dystonia 1 (CMD1). (a) Results of genome-wide association mapping of the CMD1 mutation with 12 affected animals and 14 controls using ASSHOM (red) and ASSIST (blue). The black horizontal bars mark the limits between the 29 autosomes. Evidence for linkage ( $y$ axis) is measured as $\log (1 / p)$, with $p$ being determined by 10,000 locus (ASSHOM) or phenotype (ASSIST) permutations (see Methods). (b) Genotypes of cases and controls for SNPs on chromosome 25. Homozygous genotypes are shown in yellow or white, and heterozygous genotypes in red. Overlapping blocks of extended homozygosity in the cases are marked in black and gray. The limits and size of the haplotype that is shared homozygous and identical by state among all cases is highlighted in red. (c) Above, partial gene content of the shared chromosome segment, focusing on the studied candidate genes involved in neuromuscular physiology. Middle, exonic composition of the fetal and adult forms of ATP2A1 highlighting exon 14, which harbors the mutation encoding R559C. Below, bovine sequence and interspecies alignments of the region flanking $\mathrm{R} 559 \mathrm{C}$, showing the extreme conservation of the Arg559 residue.

a

b

C animals $^{7,8}$. The incidence of RL was estimated at $0.44 \%$ in HolsteinFriesian and $2.5 \%$ in Danish Red breeds. Genealogical analysis suggests autosomal recessive inheritance ${ }^{8}$. Finally, severe forms of IF have been reported in Chianina cattle. Affected calves show cutaneous lesions, with deep fissures separating hyperkeratotic skin plaques and eversion of mucocutaneous junctions (ectropion and eclabium), reminiscent of harlequin ichthyosis in humans ${ }^{9}$.
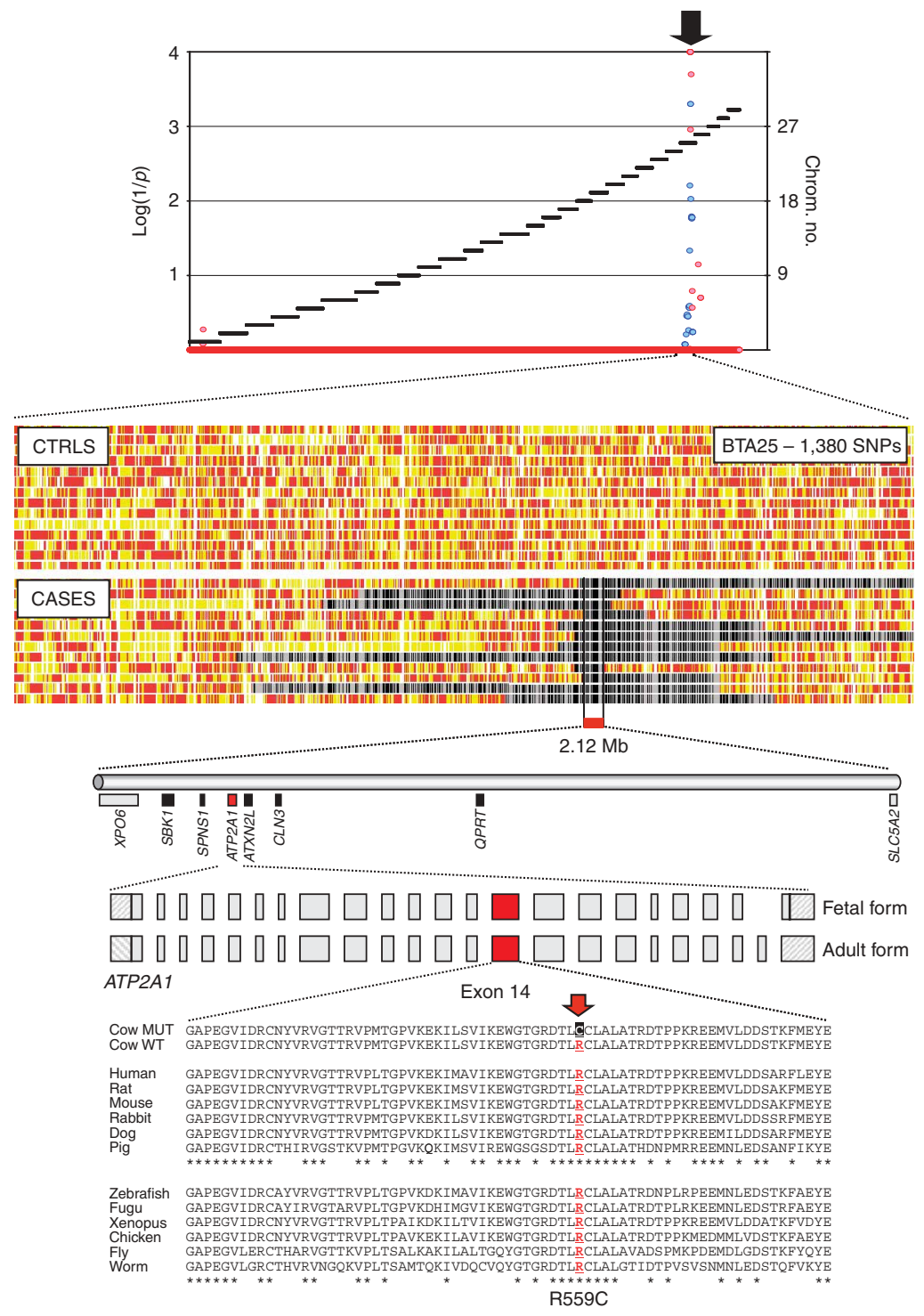
We performed genome-wide SNP scans on DNA samples from affected individuals and controls (Table 1), using either the $25 \mathrm{~K}$ Affymetrix SNP panel or a custom-made 60K Illumina panel (Supplementary Table 1 and Supplementary Fig. 1 online). The latter was assembled from SNPs in the public domain obtained from the Bovine Genome Project of the Human Genome Sequencing Center at Baylor College of Medicine and from comparing publicly available BAC end sequences with the bovine reference sequence (W. Coppieters et al., unpublished data). The assumed order of the SNP markers was based on build 4.0 of the Bos taurus genome. (See Methods for URLs for these data sources.) To map the corresponding disease loci, we developed two heuristic programs detecting overlapping, unusually long, homozygous chromosome segments among affected cattle. The first program (ASSHOM) performs homozygosity mapping by searching for shared segments of homozygosity that need not be identical by state (IBS) ${ }^{10}$. The second program (ASSIST) performs autozygosity mapping by searching for shared homozygous and IBS chromosome segments among affected cattle. Unique, genome-wide significant signals $(P \leq 0.0005)$ were readily obtained with one or both approaches for CMD1 (BTA25), CMD2 (BTA29), CTS (BTA19), RL (BTA17) and IF (BTA2). These results validated the genetic etiology of the five defects and confirmed that CMD1 and CMD2 have distinct determinants. Closer examination of the autozygous segments defined critical regions of $2.12,3.61,2.42,0.87$ and $11.78 \mathrm{Mb}$ for CMD1, CMD2, CTS, RL and IF, respectively (Table 1, Fig. 1 and Supple-

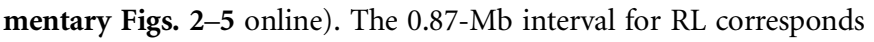
to a chromosomal segment at which all affected cattle (HolsteinFriesian and Red Danish) are homozygous for the same haplotype. This observation suggests that RL is allelically homogeneous across these breeds, and illustrates the potential gain in mapping resolution from studying the same trait across breeds, as was recently shown in the $\operatorname{dog}^{11}$.

The 2.12-Mb segment encompassing the CMD1 locus on bovine chromosome 25 encompasses 95 transcriptional units, of which 6 were plausible candidates because their protein products are known to have functions in neuromuscular physiology (Fig. 1). RT-PCR analyses in brain, spinal cord and skeletal muscle of affected individuals (cases) and controls did not reveal any striking alterations in gene expression for these genes. However, DNA sequencing revealed a missense mutation (C1676T) leading to a R559C substitution in exon 14 of
ATP2A1, which encodes a fast-twitch skeletal-muscle $\mathrm{Ca}^{2+}$ ATPase. ATP2A1 (also known as SERCA1) is responsible for pumping $\mathrm{Ca}^{2+}$ from the cytosol back into the sarcoplasmic reticulum, thereby inducing muscle relaxation ${ }^{12}$. Nonsense and splice-site mutations in the human ATP2A1 gene cause Brody myopathy (OMIM 601003), a rare autosomal recessive disorder characterized by exercise-induced muscle cramps and impaired muscle relaxation ${ }^{13,14}$. Arg559 in bovine ATP2A1 is located within the nucleotide-binding domain and is a highly conserved, functionally significant residue that directly interacts with the $\beta$-phosphate moiety of ATP (Fig. 1) ${ }^{15}$. Site-directed mutagenesis of this residue abolished ATP2A1 function ${ }^{15}$. Genotyping 2,000 unaffected Belgian Blue animals for the R559C variant did not reveal a single individual homozygous for the Cys559-encoding mutation despite a frequency of the Cys559 allele of $0.075(P=$ $\left.\left(1-0.075^{2}\right)^{2,000}=1.26 \times 10^{-5}\right)$, whereas all 81 confirmed CMD1 cases were homozygous for the mutation. Taken together, these results strongly suggest that the R559C substitution is causative for CMD1, disrupting ATP2A1 function by impairing ATP binding.

The 3.61-Mb segment containing the CMD2 locus on bovine chromosome 29 encompasses 13 genes, including one strong candidate: SLC6A5, encoding the $\mathrm{Na}^{+} / \mathrm{Cl}^{-}$-dependent glycine transporter (GlyT2), which is responsible for maintaining an abundant presynaptic pool of neurotransmitter at glycinergic synapses (Supplementary Fig. 2) ${ }^{16}$. In humans, missense, nonsense and frameshift mutations in SLC6A5 underlie autosomal recessive hyperekplexia (OMIM 149400), which is characterized by an exaggerated startle response to tactile or acoustic stimuli resulting in muscle hypertonia and life-threatening neonatal apnea episodes ${ }^{17}$, symptoms highly reminiscent of CMD2 in Belgian Blue cattle. Sequencing SLC6A5 in CMD2 cases revealed a missense mutation (T809C) in exon 4, resulting in a $\mathrm{L} 270 \mathrm{P}$ substitution in the third membrane-spanning domain of GlyT2. Although the Leu270 residue is not predicted to be involved in glycine or $\mathrm{Na}^{+}$binding ${ }^{17}$, a leucine residue is conserved at this position in all vertebrates studied, indicative of structural and functional importance (Fig. 2a,b). We did not find a single individual homozygous for the Pro270-encoding mutation among 2,000 unaffected Belgian Blue animals, despite a frequency of 0.023 for the Pro270 allele, whereas all 21 confirmed CMD2 cases were homozygous for the Pro270-encoding allele $\left(P=\left(1-0.023^{2}\right)^{2,000}=0.35\right)$. We introduced the corresponding mutation (affecting Leu269 in human a

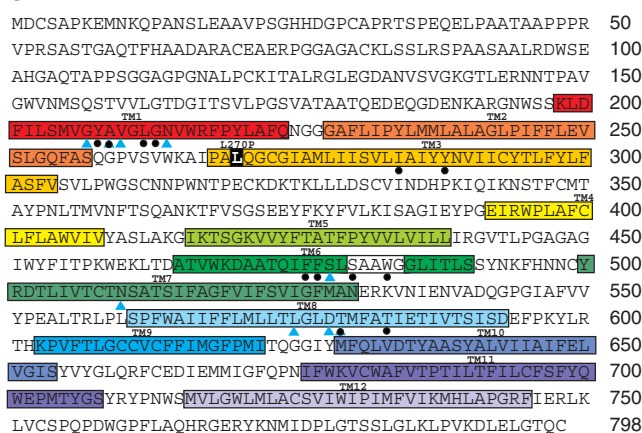

b

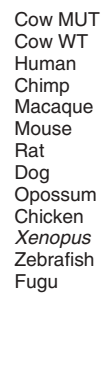

C

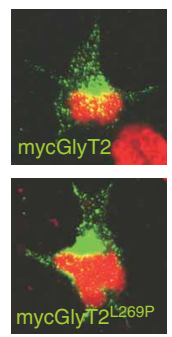

d

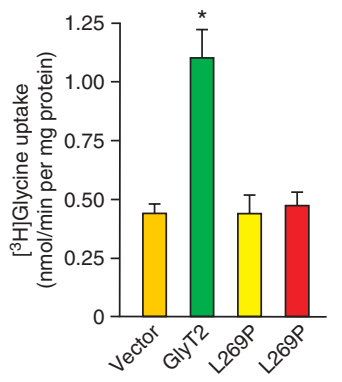

Figure 2 Congenital muscular dystonia 2 (CMD2). (a) Sequence of bovine GlyT2 protein highlighting the 12 membrane-spanning domains (TM1-12), the predicted $\mathrm{Na}^{+}$(blue triangles) and glycine (black circles) binding residues and the L270P mutation (black shading). (b) Sequence alignment of TM3 showing its remarkable conservation down to amphibians and fish, including the Leu270 residue. MUT, L270P mutant; WT, wild type. (c) Confocal images of transfected HEK293 cells showing membrane localization of myc-hGlyT2 and the myc-hGlyT2L269P mutant counterstained with the nuclear marker TOPRO3. (d) Glycine uptake in HEK293 cells transiently transfected with pRKmyc-hGlyT2 and the pRKmyc-hGlyT2L269P mutant after 5 min incubation with $200 \mu \mathrm{M}\left[{ }^{3} \mathrm{H}\right]$ glycine. Data are means \pm s.e.m. $(n=6-20)$. Statistical comparisons were made using an unpaired Student's $t$-test. *, significantly different from vector control. 
a
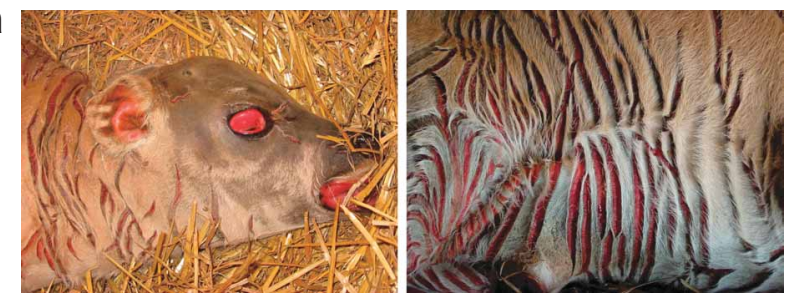

b
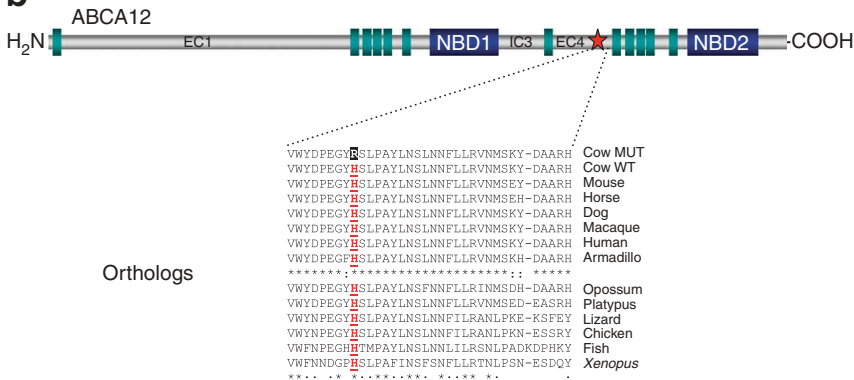

Paralogs

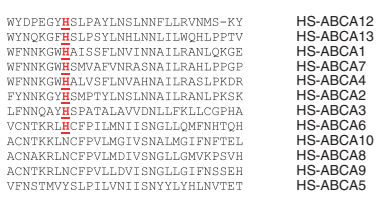

Figure 3 Ichthyosis fetalis (IF). (a) Cutaneous lesions and eversion of mucocutaneous junctions in a Chianina IF case. (b) Schematic representation of the structure of the $A B C A 12$ protein showing the position of the transmembrane helices (green) and the nucleotide-binding domains (blue). The position of the H1935R substitution associated with IF in Chianina cattle is marked by the red star. The largest extracellular (EC) and intracellular (IC) loops are numbered. Coordinates are according to ref. 25. (c) Clustal-W alignments of the amino acid sequences flanking the H1935R mutation among sequenced vertebrate $A B C A 12$ orthologs and human $A B C A$ paralogs. MUT, H1935R mutant; WT, wild type.

GlyT2) into the expression construct pRK5myc-hGlyT2 (ref. 17) by site-directed mutagenesis, and analyzed two independent mutant constructs for subcellular localization and $\left[{ }^{3} \mathrm{H}\right]$ glycine uptake in HEK293 cells. Consistent with the effect of most missense mutations in human GlyT2 (ref. 17), this substitution did not affect membrane trafficking, but abolished $\left[{ }^{3} \mathrm{H}\right]$ glycine uptake (Fig. 2c,d). Taken together, these results demonstrate that the L270P mutation is causative for CMD2 and disrupts presynaptic uptake of glycine by GlyT2.

The three IF cases available were closely related; hence, the shared segment on bovine chromosome 2 containing the IF mutation was large, $11.78 \mathrm{Mb}$ (Supplementary Fig. 3). However, we noted that the orthologous region on human chromosome 2 contains $A B C A 12$, which encodes an ATP-binding cassette $(\mathrm{ABC})$ transporter found in the lamellar granules of keratinocytes, where it is thought to have an important role in the regulation of lipid trafficking ${ }^{18}$. Missense mutations in the first ATP-binding domain of human $A B C A 12$ cause lamellar ichthyosis type 2 (ref. 19), whereas nonsense, frameshift and in rare cases missense mutations have been shown to cause the more severe harlequin ichthyosis (OMIM 242500) ${ }^{20-24}$, which markedly resembles IF. We therefore sequenced $A B C A 12$ in the affected cases and controls and identified a missense mutation in exon 39 (A5804G) resulting in an H1935R substitution in the fourth extracellular loop. The corresponding His1935 residue is conserved in the
$A B C A 12$ ortholog of all sequenced vertebrates (including amphibians and fish) as well as in 7 of the 11 human $A B C A$ transporters, underlining its key structural and functional importance (Fig. 3). Genotyping 96 healthy Chianina cattle identified two individuals that were heterozygous for the His1935- and the Arg1935-encoding alleles, whereas the three IF cases were homozygous for the Arg1935encoding allele. The Arg1935 allele was not found in other breeds $(n=60)$. Again, these findings support the disease causality of the H1935R mutation.

We have shown that using SNP arrays with a marker density on the order of one SNP per $55 \mathrm{~kb}$ allows highly effective localization of mutations causing inherited defects in livestock. It is noteworthy that neither CMD2, RL nor IF could be mapped by linkage analysis or association studies using a panel of 400 microsatellite markers and the available pedigree material (data not shown). Moreover, we show that, after having determined the map location, it is possible to rapidly identify the causal mutations in a substantial proportion of cases. Indeed, we have located the genes and mutations responsible for CMD1 (ATP2A1) and CMD2 (SLC6A5) in Belgian Blue cattle, and for ichthyosis fetalis $(A B C A 12)$ in Italian Chianina, defining new missense mutations that disrupt protein function and identifying the corresponding human disorders (Brody myopathy, hyperekplexia and harlequin ichthyosis). The 2.42- and 0-87 Mb intervals encompassing the mutations causing CTS and RL are presently being screened to identify the corresponding causative mutations (Supplementary Figs. 4 and 5).

These advances have important practical consequences, as they allow the rapid control of emerging recessive defects with otherwise major economic and animal welfare implications. This is well illustrated by the fact that the diseases CMD1 and CMD2 were essentially eliminated from the Belgian Blue breed within a matter of months after the discovery of the causal mutations. Virtually all artificial insemination sires were genotyped for both defects (revealing that $\sim 11.4 \%$ were carriers of CMD1, $4.6 \%$ of CMD2 and $0.6 \%$ of both, in good agreement with reported CMD incidence) and the genotypes published. The test was offered to breeders, allowing them to test their cows and private bulls and combine the information to avoid at-risk matings. Carrier animals are not systematically culled, as their overall breeding worth may outweigh the economic penalty of carrier status.

Domestic animal populations are a treasure trove of inherited phenotypic variability as the result of centuries of selection for useful or fashionable attributes. Our work, as well as comparable results obtained in $\operatorname{dogs}^{11}$ and undoubtedly soon to be obtained in other species, shows that the analysis of domestic animal populations has the potential to make an extremely valuable contribution toward unraveling genotype-phenotype relationships and genome function.

\section{METHODS}

Genotyping. Genotyping using the custom-made $60 \mathrm{~K}$ iSelect panel as well as the $25 \mathrm{~K}$ Affymetrix array was performed using standard procedures as recommended by the respective manufacturers. CMD1, CTS, RL and IF cases and controls were genotyped with the Illumina $60 \mathrm{~K}$ panel, whereas CMD2 cases were genotyped with the Affymetrix $25 \mathrm{~K}$ panel.

Association analysis. ASSHOM first scans the genome of each of the $N$ studied cases for blocks of adjacent homozygous markers. Markers within such blocks receive a score $s_{i j}$ corresponding to the sum of $-\log \left(p_{i}^{2}\right)$ over all markers in the block. In this, $p_{i}$ is the frequency (estimated in controls) of the allele for which case $i$ is homozygous and $j$ is the marker number (out of $M$ markers). Thus, the longer and rarer the homozygous haplotype, the higher the score. All markers 
for which individual $i$ is heterozygous receive the same $10^{-5}$ score. ASSHOM then computes a summary score at each marker position, $S_{j}$, corresponding to the harmonic mean of the $N$ corresponding $s_{i j}$ scores. If one or more of the cases are heterozygous at the corresponding marker position, their $10^{-5}$ score will drastically penalize $S_{j}$. The statistical significance of each $S_{j}$ is estimated from its rank with respect to a list of 'best out of $M$ ' $P_{j}$ scores calculated by 'locus permutation'. Locus permutation scores $P_{j}$ are computed as the harmonic mean of $N$ randomly selected $s_{i j}$ scores (one per case). The corresponding $P$ values are thus corrected for the testing of $M$ marker loci and account for the level of inbreeding of the cases. ASSIST scans the genome for core markers defined as polymorphic in controls yet monomorphic in cases. For each core marker, ASSIST identifies a proximal reference case with the longest contiguous stretch of homozygous markers proximal from the core, and a distal reference case with the longest contiguous stretch of homozygous markers distal from the core. Proximal (or, respectively, distal) scores are then computed as the sum of $-\log \left(p_{i}^{2}\right)$ over contiguous (starting from the core) marker stretches shared IBS with the proximal (respectively, distal) reference. In this, $p_{i}$ is the frequency in controls of the allele for which the reference case is homozygous. Proximal and distal scores are summed to yield an overall score $S_{j}$. Their statistical significances are determined by 'phenotype permutation': disease status is randomly redistributed amongst cases and controls, genomewide association conducted with ASSIST and the 'best out of $M$ ' phenotype permutation scores $P_{j}$ scores stored. The $P$ value of real $S_{j}$ s are then estimated from their rank with respect to a list of such 'best out of $M^{\prime} P_{j}$ permutation scores. ASSIST thus accounts for multiple-marker testing and allelic frequencies in controls.

Both programs assume that the analyzed trait has simple, monogenic recessive inheritance (incomplete penetrance is not accommodated). When samples originating from distinct breeds are analyzed together, ASSHOM assumes allelic homogeneity within but not necessarily between breeds, whereas ASSIST assumes allelic homogeneity both within and between breeds. ASSHOM and ASSIST are available from the authors on request.

Resequencing. For each disease, at least one affected animal and an unrelated wild-type counterpart, matched for age and breed, were chosen after careful phenotyping. They were slaughtered following ethical procedures. Total blood was withdrawn for DNA extraction and tissues samples were collected directly after the animal's death, rapidly frozen in liquid nitrogen and stored at $-80{ }^{\circ} \mathrm{C}$ until RNA extraction. The tissue panel was disease specific: skeletal muscle, spinal cord and brain for CMD1 and CMD2; cartilage, skeletal muscle and spinal cord for CTS; kidney and skeletal muscle for RL; and skin for IF.

Total RNA was extracted from tissue samples after homogenization in TRIzol Reagent (Invitrogen) following the manufacturer's instructions. Total RNA was ethanol precipitated, resuspended in DEPC-treated water and diluted at $1 \mu \mathrm{g} / \mu \mathrm{l}$. First-strand cDNA was synthesized with the Superscript First-Strand Synthesis System for RT-PCR (Life Technologies) using a mixture of random hexamers and oligo(dT) primers. The cDNA was directly amplified by PCR with gene-specific primers designed to allow full-length ORF amplification. Primer pairs and PCR conditions for all the positional candidate genes screened are listed in Supplementary Table 2 online. PCR products were loaded on 1\% agarose gels to compare band intensities and sizes between affected and control animals. DNA fragments were cut and purified with the GENECLEAN Kit (Qbiogene). Purified fragments were sequenced using the Big Dye terminator cycle-sequencing kit (Applied Biosystems). Electrophoresis of purified sequencing reactions was performed on an ABI PRISM 3730 DNA analyzer (PE Applied Biosystems). Multiple sequence traces from affected and wildtype animals were aligned and compared using the phredPhrap program from the Consed package. Pathogenic relevance of each mutation was established by aligning the protein sequence from available species.

Genomic DNA was extracted from total blood through a standard phenolchloroform extraction procedure. Each animal enrolled for the resequencing study was first genotyped for SNP markers from the minimum genetic interval to asses its IBD (affected) or non- IBD (wild-type counterpart) status. Finally, all the available individuals (Table 1) were genotyped for the respective disease mutations by PCR amplification of the culprit exon followed by resequencing. Primer pairs for each mutation are listed in Supplementary Table 2.
Functional analysis of GlyT2. $\left[{ }^{3} \mathrm{H}\right]$ Glycine uptake assays were performed as described ${ }^{17}$ in HEK293 cells (ATCC CRL1573) transfected with $0.25 \mu \mathrm{g}$ total pRK5myc (vector control), pRK5myc-hGlyT2 or the L269P mutant using Lipofectamine LTX (Invitrogen). After $24 \mathrm{~h}$, cells were washed with a buffer containing $116 \mathrm{mM} \mathrm{NaCl}, 1 \mathrm{mM} \mathrm{NaH}_{2} \mathrm{PO}_{4}, 26 \mathrm{mM} \mathrm{NaHCO}_{3}, 1.5 \mathrm{mM} \mathrm{MgSO}_{4}$, $5 \mathrm{mM} \mathrm{KCl}, 1.3 \mathrm{mM} \mathrm{CaCl}_{2}$ and $5 \mathrm{mM}$ glucose pregassed with $5 \% \mathrm{CO}_{2} / 95 \%$ air and then incubated for $5 \mathrm{~min}$ in $1 \mu \mathrm{Ci} / \mathrm{ml}\left[{ }^{3} \mathrm{H}\right]$ glycine $(60 \mathrm{Ci} / \mathrm{mmol}, \mathrm{NEN})$ at a final concentration of $200 \mu \mathrm{M}$. Uptake was terminated by two additions of icecold buffer followed by aspiration. Cells were digested in $0.1 \mathrm{M} \mathrm{NaOH}$ and used for scintillation counting and determination of protein concentration using the Bradford reagent (Bio-Rad). $\left[{ }^{3} \mathrm{H}\right]$ Glycine uptake was calculated as $\mathrm{nmol} / \mathrm{min}$ per mg protein and expressed as a percentage of the pRK5myc-transfected control. All statistical comparisons used an unpaired Student's $t$-test. For GlyT2 immunostaining, transfected cells were washed twice in PBS, fixed for $5 \mathrm{~min}$ in $4 \%(\mathrm{w} / \mathrm{v})$ paraformaldehyde in PBS and quenched in $50 \mathrm{mM} \mathrm{NH}_{4} \mathrm{Cl}$ for $10 \mathrm{~min}$ before being permeabilized in $0.01 \%(\mathrm{w} / \mathrm{v})$ Triton X-100, further washed in PBS and stained with an anti-Myc primary antibody and Alexa Fluor 488conjugated secondary antibodies (Invitrogen). Confocal microscopy was performed using a Zeiss Axioscop LSM 510 Meta confocal microscope with $\times 40$ objective (NA 1.3) and $\times 3$ digital zoom, capturing images at $1,024 \times 1,024-$ pixel resolution.

URLs. 25K Affymetrix bovine SNP panel, http://www.affymetrix.com/ products/arrays/specific/bovine.affx; Bovine Genome Project of the Human Genome Sequencing Center at Baylor College of Medicine, including build 4.0 of the Bos taurus genome, http://www.hgsc.bcm.tmc.edu/projects/bovine/.

Note: Supplementary information is available on the Nature Genetics website.

\section{ACKNOWLEDGMENTS}

The $60 \mathrm{~K}$ genome-wide SNP panel was constructed with the financial support of Holland Genetics (Arnhem, The Netherlands). This work was funded by grants from the Walloon Ministry of Agriculture, from the Belgian Science Policy organization (SSTC Genefunc PAI), from the Communauté Française de Belgique (Game \& Biomod ARC), from the University of Liège and from the Medical Research Council (G0601585 to K.H. and R.J.H.). C.C. is Chercheur Qualifié from the Fonds National de la Recherche Scientifique. This work is dedicated to the memory of Professor Roger Hanset.

\section{AUTHOR CONTRIBUTIONS}

C.C. and M.G. designed the experiments, analyzed data, wrote the manuscript and supervised the project. W.C. designed the Illumina iSelect panel and supervised genotyping. F.R., D.D., J.S.A., M.D., J.-C.F., R.H., C.J., S.V., M.L. and M.F. confirmed diagnosis and provided samples of affected individuals. S.L. and M.K. performed genotyping on the Affymetrix panel. N.C., S.D., C.F., X.H., L.K., P.S., N.T. and H.N. performed genotyping and mutation scanning. X.H. analyzed data and mapped the CMD1 mutation. C.J. and M.F. performed mutation scanning of RL. E.C., K.H., B.R.P. and R.J.H. performed functional test of the CMD2 mutation. R.J.H. participated in writing the manuscript.

Published online at http://www.nature.com/naturegenetics

Reprints and permissions information is available online at http://npg.nature.com/ reprintsandpermissions

1. Young, C.W. \& Seykora, A.J. Estimates of inbreeding and relationship among registered Holstein females in the United States. J. Dairy Sci. 79, 502-505 (1996).

2. Hayes, B.J., Visscher, P.M., McPartlan, H.C. \& Goddard, M.E. Novel multilocus measure of linkage disequilibrium to estimate past effective population size. Genome Res. 13, 635-643 (2003).

3. Shuster, D.E., Kehrli, M.E. Jr., Ackermann, M.R. \& Gilbert, R.O. Identification and prevalence of a genetic defect that causes leukocyte adhesion deficiency in Holstein cattle. Proc. Natl. Acad. Sci. USA 89, 9225-9229 (1992).

4. Thomsen, B. et al. A missense mutation in the bovine SLC35A3 gene, encoding a UDP$\mathrm{N}$-acetylglucosamine transporter, causes complex vertebral malformation. Genome Res. 16, 97-105 (2006).

5. Pierce, K.D. et al. A nonsense mutation in the alpha 1 subunit of the inhibitory glycine receptor associated with bovine myoclonus. Mol. Cell. Neurosci. 17, 354-363 (2001).

6. Vandeputte, S. et al. Congenital muscular dystonia (CMD): a new congenital pathology in Belgian Blue calves. in Proceedings of the 24th World Buiatrics Congress, Nice, 15-19 October 2006 (ref. OS05-2).

7. Agerholm, J.S. Inherited disorders in Danish cattle. APMIS Suppl. 122, 1-76 (2007). 
8. Rude, H., Agerholm, J.S., Maddox-Hyttel, P., Christensen, K. \& Flagstad, P. Renal lipofuscinosis in Danish slaughter cattle. J. Comp. Pathol. 132, 303-312 (2005).

9. Molteni, L. et al. Ichthyosis in Chianina cattle. Vet. Rec. 158, 412-414 (2006).

10. Lander, E.S. \& Botstein, D. Homozygosity mapping: a way to map human recessive traits with the DNA of inbred children. Science 236, 1567-1570 (1987).

11. Karlsson, E.K. et al. Efficient mapping of mendelian traits in dogs through genomewide association. Nat. Genet. 39, 1321-1328 (2007).

12. Toyoshima, C. \& Inesi, G. Structural basis of ion pumping by $\mathrm{Ca}^{2+}-$ ATPase of the sarcoplasmic reticulum. Annu. Rev. Biochem. 73, 269-292 (2004).

13. Odermatt, A. et al. Mutations in the gene encoding SERCA1, the fast-twitch skeletal muscle sarcoplasmic reticulum $\mathrm{Ca}^{2+}$ ATPase, are associated with Brody disease. Nat. Genet. 14, 191-194 (1996).

14. Berchtold, M.W., Brinkmeier, H. \& Müntener, M. Calcium ion in skeletal muscle: its crucial role for muscle function, plasticity, and disease. Physiol. Rev. 80, 1215-1265 (2000).

15. Ma, H., Lewis, D., Xu, C., Inesi, G. \& Toyoshima, C. Functional and structural roles of critical amino acids within the "N", "P", and "A" domains of the $\mathrm{Ca}^{2+}$ ATPase (SERCA) headpiece. Biochemistry 44, 8090-8100 (2005).

16. Gomeza, J. et al. Deletion of the mouse glycine transporter 2 results in a hyperekplexia phenotype and postnatal lethality. Neuron 40, 797-806 (2003).
17. Rees, M.I. et al. Mutations in the human GlyT2 gene define a presynaptic component of human startle disease. Nat. Genet. 38, 801-806 (2006).

18. Akiyama, M. Pathomechanisms of harlequin ichthyosis and ABCA transporters in human diseases. Arch. Dermatol. 142, 914-918 (2006).

19. Lefévre, $\mathrm{C}$. et al. Mutations in the transporter $A B C A 12$ are associated with lamellar ichthyosis type 2. Hum. Mol. Genet. 12, 2369-2378 (2003).

20. Kelsell, D.P. et al. Mutations in ABCA12 underlie the severe congenital skin disease harlequin ichthyosis. Am. J. Hum. Genet. 76, 794-803 (2005).

21. Akiyama, M. et al. Mutations in lipid transporter $A B C A 12$ in harlequin ichthyosis and functional recovery by corrective gene transfer. J. Clin. Invest. 115, 1777-1784 (2005).

22. Thomas, A.C. et al. $A B C A 12$ is the major harlequin ichthyosis gene. J. Invest Dermatol. 126, 2408-2413 (2006).

23. Akiyama, M. et al. Compound heterozygous $A B C A 12$ mutations including a novel nonsense mutation underlie harlequin ichthyosis. Dermatology 215, 155-159 (2007).

24. Thomas, A.C. et al. Novel and recurring $A B C A 12$ mutations associated with harlequin ichthyosis: implications for prenatal diagnosis. Br. J. Dermatol. 158, 611-613.

25. Peelman, F. et al. Characterization of the ABCA transporter subfamily: identification of prokaryotic and eukaryotic members, phylogeny and topology. J. Mol. Biol. 325, 259-274 (2003). 